\title{
A Hybrid Technique for Tunneling Mechanism of IPv6 using Teredo and 6RD to Enhance the Network Performance
}

\author{
Zulfiqar Ali Zardari ${ }^{1}$ \\ Faculty of Information Technology \\ Beijing University of Technology \\ Beijing, China
}

\author{
Munwar $\mathrm{Ali}^{2}$ \\ Department of Software Engineering. MUET SZAB \\ Khairpur Mirs', Pakistan
}

\author{
Reehan Ali Shah ${ }^{3}$ \\ Computer System Engineering \\ University College of Engineering \& Technology (UCET) \\ The Islamia University Bahawalpur
}

\author{
Ladha Hussain Zardari ${ }^{4}$ \\ Department of Information Technology, QUEST, \\ Nawabshah, Pakistan
}

\begin{abstract}
Currently, Internet Protocol version 4 (IPv4) addresses have been depleted. Many Internet Service Providers (ISPs), researchers and end users are migrating from IPv4 to IPv6 due to strong features of IPv6 and limitation in IPv4. Different tunneling techniques have been deployed to migrate on IPv6 for ordinary users. However, these techniques create many issues such as compatibility, complexity, connectivity and traffic. Due to the dissimilar header structure and incompatibility of IPv4 and IPv6, the devices are unable to communicate with each other because devices do not support IPv6 addresses directly. The performance of network is also compromised due to huge increment in data transmission traffic. In this paper, we proposed a technique to provide full IPv6 connectivity and enhancing the network performances by combining two tunneling techniques such as IPv6 Rapid Deployment (6RD) and Teredo. To increase the throughput of the network, jumbo frames are used to carry huge amount of data. The main objective of using both techniques is to provide a hybrid network rendering for full IPv6 connectivity. The proposed technique provides not only the IPv6 but also provides better performance in the network. Simulation results show that throughput and packet delivery ratio achieve maximum gain by 9000 bytes and $98 \%$, respectively.
\end{abstract}

Keywords-Hybrid network; IPv4; IPv6; 6RD; teredo tunneling mechanism; network performance jumbo frames

\section{INTRODUCTION}

In February 2011, internet assigned number authority (INNA) declared that there are no IP addresses available on IPv4 pool. After this announcement the shortfalls of IP addresses create problems to provide connectivity for end users. In such condition the only solution is to migrate on IPv6 which contains trillion of trillion IP addresses (128 bits). It is not an easy task to shift from IPv4 to IPv6 quickly but a big challenge of next generation of internet. Many translation, transitions and tunneling techniques are available such as IPv4In-IPv6 tunneling, Teredo, 6to4, dual stack and other. These methods are planned to extend IPv6 connectivity using IPv4 network [1-4]. But out of these two techniques Teredo and
6RD are widely used by research community. This research focuses on both Teredo and 6RD.Currently, illustrated issue is the connectivity of IPV4 and IPV6 under the same environment. Devices are not upgraded to support IPv6 connectivity and Compatibility. There is a need of hybrid network so that IPv4/IPv6 can communicate across both the network infrastructure. It is difficult for ISPs to control the flow of packets in 6to 4 tunnel prefix from customers for this reason 6RD is proposed. The rapid use of social networks and multimedia application create high data transmission load and cause hindering the traffic to raise the problem that how to move this traffic on IPv4 network. The jumbo frames has high capacity and is specially designed to carry heavy load of traffic in the network [5-7].

This paper focuses on deployment of IPv6 with IPv4 network. The proposed work merge Teredo and 6RD together to create a hybrid network. The features of both networks are combined to facilitate the current IPv4 addresses, the issues of 6RD can be covered in Teredo and vice versa. IPv6 have a unique feature of IPsec and huge amount of IPs over IPv4 [8]. Proposed hybrid network provides full connectivity to customers/ISPs using these two techniques. After deployment of hybrid network the performance of the overall network is highly reliable.

The contributions of this proposed work are: (1) an adaptation of an improved hybrid-based IPv6 mechanism is deployed, which enables IPv6 (2) Examination and illustration of IPv6 development system is performed incorporating traffic characteristics tunneling. (3) A solution is provided to handle the increased payload. (4) Achieved results for packet delivery ratio in hybrid network jumbo frame are included. This approach facilitates the Internet service providers (ISPs), companies and vendors in migration of IPv4 and especially for those ISPs having large network across the any province or country.

The rest of the paper is divided into following parts, section 2 describes the related work, section 3 is about problem 
statement, section 4 is methodology, section 5 covers the simulations and results, and section 6 is about conclusion.

\section{RELATED WORK}

Ala Hamarsheh et al [9] proposed mechanism for known as D4across6. It consists of deployment of IPv4 locally for IPv6 connectivity to access network. Mechanism allows IPv4 devices to communicate with IPv4-only content providers over an IPv6-only infrastructure. It carries IPv4 packets and routing information of the IPv4 protocol over an IPv6-only network infrastructure. The features of the proposed protocol are: offers IPv4 connectivity across IPv6-only access networks, stateless operation, cost-effective solution, and requires simple and automatic configuration at customers' hosts and no change in network infrastructure is needed. Results are calculated in one parameter throughput which varies from 64 bytes to 1024 bytes with number of nodes 100 to 1500 , while other parameter is Mbps which is dynamically changes as number of bytes are increasing. Compared with 4rd, $(\mathrm{A}+\mathrm{P})$, DS-Lite, D4across6 and it shows that performance of D4across6 is comparatively is better all other techniques. The main drawback of this is mechanism it can't handle huge amount of traffic load, whenever traffic increases it may takes more time transfer the data packets.

Fatema Siddika et al [10] proposed a solution to migration IPv6 in Bangladesh by applying transition mechanism to provide connectivity to IPv6 hosts. Specific routing protocols are implemented in networks i.e. Border Gateway Protocol (BGP), Open Shortest Path First (OSPF), and OSPFv3 for addressing and connectivity of end nodes. The proposed solution is divided into three phases first, apply a transition mechanism to the IPv4 dominant network to provide IPv6 support and make it an IPv6 dominant network. Second, apply the transition mechanism to the IPv6 dominant network to provide IPv4 support until the remaining IPv4 hosts became IPv6 hosts. Third, the network is completely in IPv6. Prevent different nodes in the network from talking with each other with different IP protocols, if necessary, upgrade the applications or use the proxy at application layer. The results carried out with that dual stack tunneling techniques deployed in this network gains better performance than others.

Shaneel Narayan et al [11] proposed a solution for transferring high traffic load one to end to another. This scheme utilizes jumbo frames to transfer the data packets during communication. This can be achieved by connecting two powerful systems and two network card (windows server) with each other. Compare the performance of jumbo frames to that of normal frames on a test-bed. To do so, two different operating systems were implemented with both IPv4 and IPv6 and it has been shown that jumbo frames undoubtedly give higher throughput than normal frames. When used on networks with IPv6, favorable delay values are attained and packet drop rates are generally better than that on IPv4 implementation. Jumbo frames overall give better network performance metrics than when normal frames are implemented on a network.

Zhao Qin et al [12] proposed seamless converging system for IPv4/IPv6 transition which is based on two parts zone-1 state full and zone-2 stateless protocol modules. It can share to addresses of IPv4 or IPv6 to access IPv4 connectivity across
IPv6 network. In zone-1 is designed as Metropolitan Area Network (MAN) in IPV6 addresses and (MAN) is also designed for IPv4 addresses. In zone-2 core routers (CR) are placed of both addresses are connected with state full translator. In zone- $2 \mathrm{CR}$ router of IPv4 connected with stateless of zone-1, in this both zone are communicating each other. Service Router (SR) and Broadband Access Server (BAS) of both networks IPv4 and IPv6 are placed to transfer data packets. It supports connectivity of IPv6 quickly, additionally it supports both addresses simultaneously without affecting each other.

S. Aravind et al [13] proposed dual stack and tunneling techniques for migration of IPv6 to transmit data packets of IPV addresses to IPv4 in both scenarios i.e. static and dynamic of the network. In the network five routers and four switches are placed in simulation for transferring data packets between both the networks. Using Graphical Network Simulator-3 (GNS3) network simulator the results are carried in dynamic, static routing network and Open Shortest Path First (OSPF) protocols to transmit IPv6 packets on IPv4 network. Performance comparison shows that low Latency and high throughput in IPv6 with respect to IPv4.

M. F. Suleiman et al [14] has point out the factor, challenges and analyzed the network by deployment of IPv6 address in Nigeria. In first phase the author survey the IPv6 awareness training, planning and deployment of IPv6. After successfully deployment of IPv6 addresses they have expressed the results and finally phase simulations experiments to explains the whole mechanism of dual stack and 6to 4 techniques in detail. A real time business case was the discussed to motivate and promote the benefits, cost estimation, risk of IPv6 addresses.

S. Javid et al [15] implemented OSPFv3 in IPv6 addresses to survival of IPs because of various features of OSPFv3 protocol. OSPFv3 is used for large and complex network to provide IPv6 connectivity to end users. The migration of IPv6 is in slow process it may takes long time to fully migrate on IPv6. The reason behind implementation is big organizations are fully depended on internet and they don't want to bear any loss if any time their services can be downtime. So avoid this issue the author has implemented to provide full connectivity to large organizations.

\section{PROBLEM STATEMENT}

Nowadays IPv6 infrastructure is a necessity of internet service providers. However some challenges, difficulties and delusion are the major concerns during deployment. These concerns are enlisted below:

- ISPs below Tier-3 are not IPv6 supported

- Deployment of IPv6 takes long time

- Replacement of equipment is a big challenge

- Unavailability of IPV6 supported devices on vender end

- Payload increases in the network

- People are irrevocable and reluctant to IPv4 and IPv6 respectively 
- At this time migration can be done slowly and gradually, although IPv6 is in practical form but still it takes long time to put into operations. [16, 17, 18]

Keeping above stated reasons in consideration migration can be done slowly and, although IPv6 is in practical form but still it takes long time to put into operations. The transition techniques used for IPv6 connectivity are summarized in Fig. 1.

The following Fig. 1 shows the hierarchy of transition which shows the different layer there are various transition techniques are used for IPv6 connectivity.

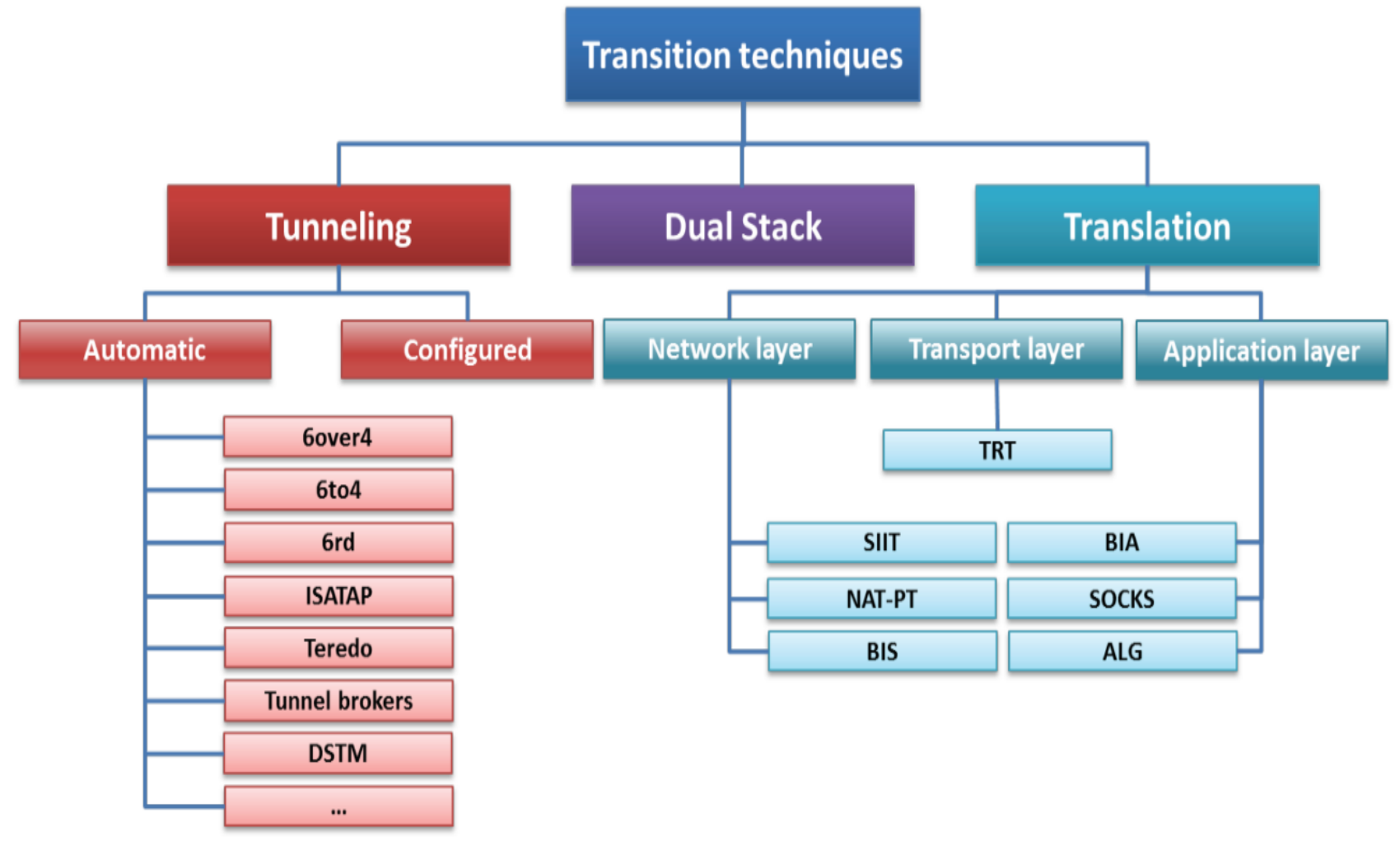

Fig. 1. Summarization of different transition techniques

In Fig. 1 transitions techniques are divided into three main techniques, tunneling, dual stack and translation. In tunneling automatic techniques are widely used for communication between IPv4 and IPv6.Internet service provider (ISP) configures tunneling and translation techniques to providing IPv6 connectivity in real time environment [19].

The research finding and contributions of this work presented as follows:

- Propose a hybrid technique based on 6RD and Teredo, which provides relieve IPv4 addresses shortage.

- The proposed hybrid technique delivers an introduction of IPv6 addresses.

- The proposed technique gives an easy way to migrate from IPv4 to IPv6 addresses

- The hybrid technique is capable of running both addresses on the same network without affecting each other.

\section{PROPOSED TECHNIQUE}

After study of all mechanisms their advantages and disadvantages in mind we have proposed a technique which is based on hybrid network that is the combination of two techniques which are Teredo \& 6RD, used to transfer data from IPv4 to IPv6. The proposed hybrid technique supports both networks IPv4 and IPv6. It can provides IPv4 addresses as well as IPv6 addresses. The hybrid network contains 4 Cisco routers connected with IPv6 hosts, IPv4 backbone network of 6RD \& Teredo relay devices. The traffic moves from IPv6 to IPv4 network via these connected devices. Addresses of IPv4 \& IPv6 are configured at every router and tunnel is configured on the boarder routers to provide the access of both networks. It is unable to provide the IPv6 connectivity to users without boarder routers because one can't send the addresses of IPv6 directly. When huge amount of data come over the network it might be some interruption in the network. By the use of jumbo frames, throughput can be increased, because it can carry data up to 9000 Bytes in the network. The proposed hybrid network a whole traffic of IPv6 is divided into two parts that are local traffic and tunneled traffic. The UPD and TCP protocols are used to encapsulate the tunneled traffic. To provide the connectivity of IPv6 behind the NAT devices is responsible to is responsibility of Teredo. It depends on two main components that are Teredo relay and Teredo Server. Teredo Server is used to initialize the Teredo, while relays are used for routing connectivity of IPv4 and IPv6. The upper level 
contain IPv6 network and lower level has IPv4 network pool. The BR router and Teredo Server functions like bridge between IPv4 and IPv6 networks. Native traffic routed by IPv6 host is encapsulated in IPV4 by CE router and forwarded towards the $\mathrm{BR}$ router or directed to $6 \mathrm{rd}$. The received encapsulated 6rd and BR traffic is de-capsulated by $\mathrm{CE}$ router and forwarded to the end user. Server assist client to get IP from IPV6 for the internet access. IPv6 packets are encapsulated in to IPv4 UDP Teredo client address by using Teredo relay and vice versa. The 3FFE:831F::/32 IPv6 suffix is advertised by Teredo relay for internet. Different IPv6 hosts send the packets to Teredo client routed by Teredo relays to share dynamic load over every Teredo client. Fig.2 shows hybrid network technique, the communication link between two terminals 6RD and Teredo techniques. The IPv6 traffic flows from upper section of the network, after that decapsulated on the edge router to the next endpoint crossing IPv4 network. To ensure the security of data packets thorough tunneled traffic is needs to be de-capsulated.

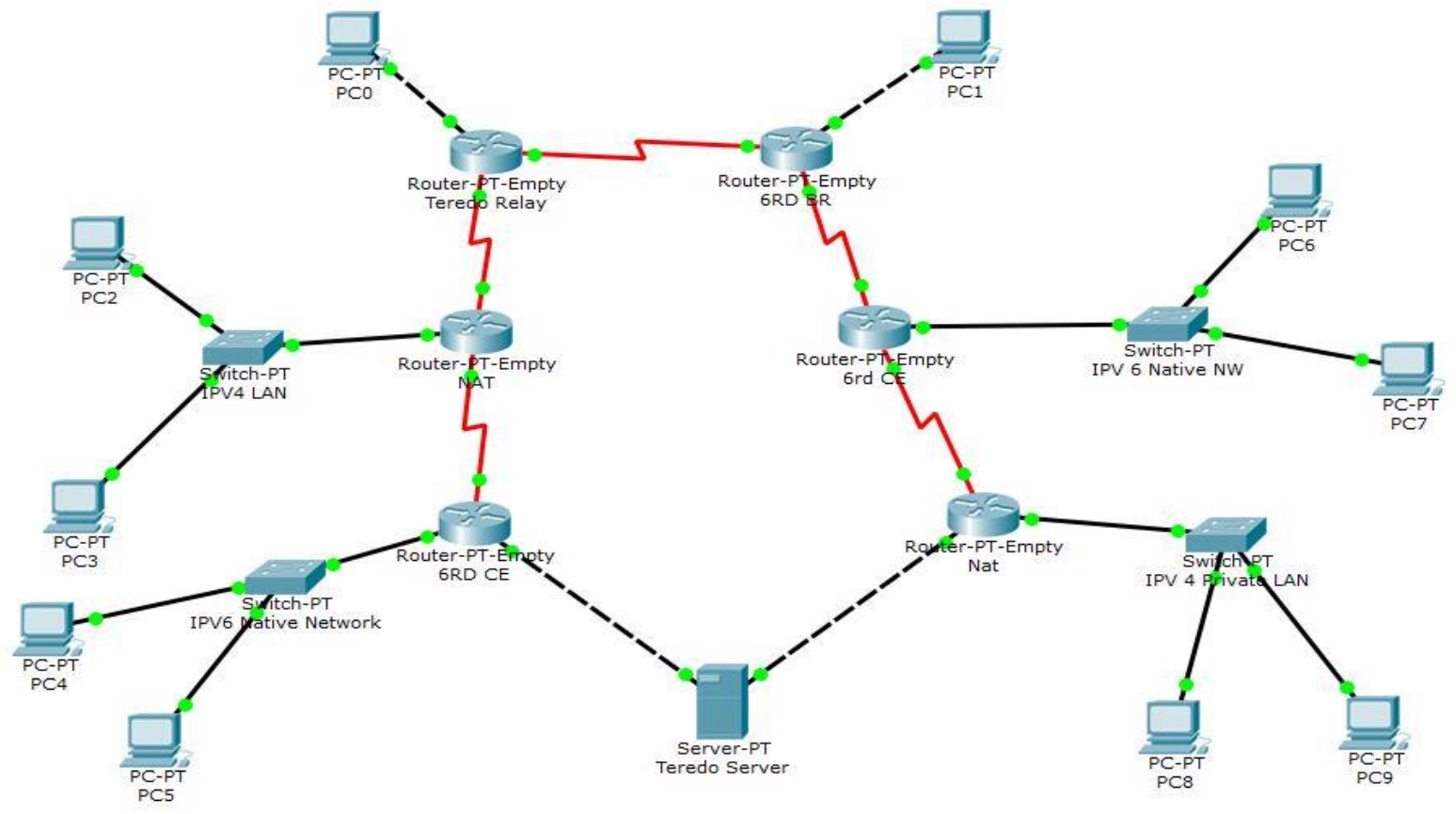

Fig. 2. Hybrid Network topology

\section{Simulations AND Result ANALYSiS}

In our proposed hybrid technique we have deployment IPv4 and IPv6 addresses successfully. In order to check the performance of the hybrid network tests are conducted amongst IPv4 \& IPv6 to show the connectivity of devices results. Simulation work scenario is created using graphical network simulator 3 (GNS3). Results analysis is carried out for evaluation among the performance metrics of these strategies in order to estimate any statistically-important variation between them. The main purpose of this research is to rank the IPv6 transition strategies and categorize the better strategy that offers high throughput, high packet delivery ratio and lowest delay. This section presents the simulation results by comparing different parameters like throughput, packet delivery ratio, average delay, tunneling overhead and jitter. Proposed network demonstrate the bundle conveyance proportion in cross breed network. Hybrid network connected with core of Teredo and 6RD routers along with IPv6 do not require header checksum, flags and identifier therefore, the IPv6 performs faster than other counterparts. Initially the throughput is very low because of less number of packets transferred as the number of packets increases throughput also increased. When the jumbo frames are used in the network it is favorable for increased throughput and packet delivery ratio as compare to 6RD and Teredo technique. The following subsections discusses these analyzed parameters and obtained results in more details. Configuration of GNS3 is easy to deploy because it shows the network graphically.GNS3 allows to access cisco devices virtually, does not required any physical hardware for simulation. The front end of GNS3 dynagen. Dynagen runs dynampis to create simulation environment. Actually GNS3 access cisco IOSs on your windows or linux systems to design network topology virtually without hardware.

\section{A. Throughput}

Fig.3 shows the throughput of IPv4 and IPv6 networks. Initially, both networks are sound and safe, however when the number of packets increases they effects throughput directly. Initially both networks i.e. IPv4 and IPv6 are smooth but as soon as packet size increases simultaneously traffic will increased. It was observed that consequent the network to stability due to jumbo frame takes the loads from the network. It is clearly seen that IPv4 and IPv6 provides better performance by the utilization of hybrid network. 


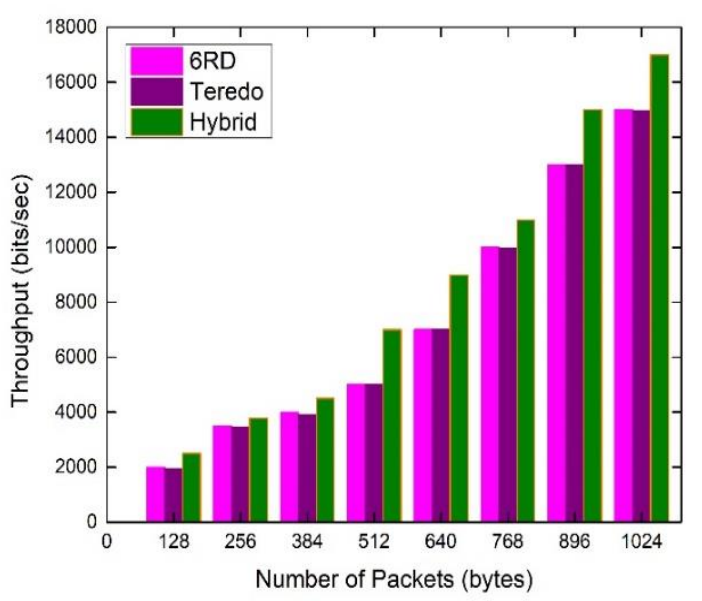

Fig. 3. Throughput of $6 \mathrm{RD}$, Teredo and Hybrid

\section{B. Packet Delivery Ratio (PDR)}

Hybrid network shows better performance in the packet delivery ratio, because the insertion of jumbo frames in the network for high payload carried out the network traffic to the destination. It is observed that with the increase of packet size PDR increases. This effect is resulted due to jumbo frames have capability to carry high traffic load to make network performance better Fi. 4 mimics this effect.

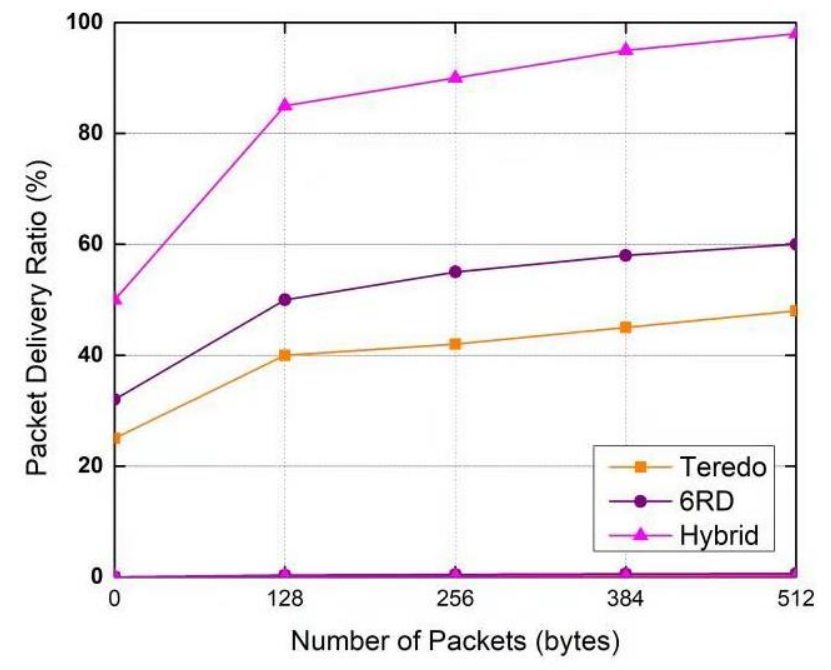

Fig. 4. Packet deliver ratio of 6RD, Teredo and Hybrid

\section{Average Delay}

Fig.5 shows the average delay with respect to time, IPv6 increases delay at end stages and that effect is carried from many devices. IPv6 shows higher delay as compared to IPv4 from initial time. While passing the traffic from one end to another node, many devices face high payload on every node in the network. Teredo clients devices are located behind the NAT devices and traffic pass through connecting server and encapsulates the incoming the packet and de-capsulate each packet before sending therefore it takes some time.

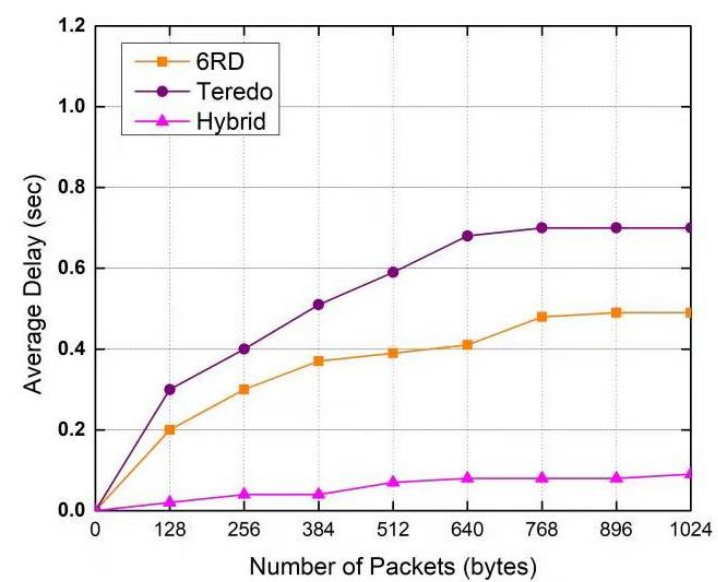

Fig. 5. Average delay of 6RD, Teredo and Hybrid

\section{Tunneling Overhead}

Fig.6 shows the sum of the overhead caused by engendering tunnels, expunging tunnels, stimulating tunnels, encapsulation, and de-capsulation is called tunneling overhead. Fig. 6 shows the tunneling overhead of 6RD, Teredo and proposed technique. It is measured with respect to packets in bytes. In average overhead it clearly declares that our proposed hybrid technique supersede both the Teredo and 6RD mechanism and hence can be proposed for complex networks to reduce the overhead drastically.

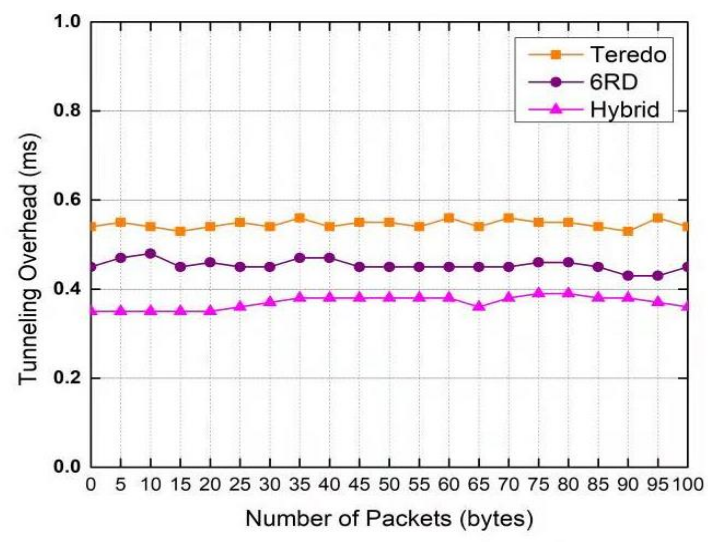

Fig. 6. Tunneling Overhead 6RD, Teredo and Hybrid

\section{CONCLUSION}

This paper provides two main contributions, connectivity of IPv6 to end users and transfer a huge amount of data packets (high payload). Two testbeds are used i.e. 6RD and Teredo to performance tunneling mechanism and to develop a hybrid network. The proposed hybrid network contains characteristic of both techniques. It has been analyzed that our proposed hybrid network has better performance than individual techniques. 6RD \& Teredo failed to provide IPv6 connectivity to the individual user. The main advantage of a hybrid network is it can carry out both networks IPv4 and IPv6 together. Both networks performance quite satisfactory and operate easily without any disturbances. Additionally, huge traffic can be transferred across the network with jumbo frame technique. 
The proposed hybrid network is compared with 6RD, and Teredo in terms of throughput, packet delivery ratio, average delay and tunneling overhead. From results, it has been observed that the hybrid network has better performance than 6RD and Teredo in term of packet delivery ratio up to $98 \%$, whereas minimum delay occurs at high payload. Collectively, the hybrid network gives a huge improvement in different metrics. For future work, this research can be extended in the perspective of security issues and different security algorithms can be developed to prevent the hybrid network from different attacks.

\section{REFERENCES}

[1] R. Tadayoni, and A. Henten, 'From IPv4 to IPv6: Lost in translation?', In Telematics and Informatics, vol. 33, year 2016 Issue 2, pp 650-659.

[2] L. Smith, M. Jacobi and S. Al-Khayatt, "Evaluation of IPv6 transition mechanisms using QoS service policies", 2018 11th International Symposium on Communication Systems, Networks \& Digital Signal Processing (CSNDSP), Budapest, Hungary, 2018, pp. 1-5.

[3] M. Nikkhah, "Maintaining the progress of IPv6 adoption", In Computer Networks, vol 102, 2016, pp 50-69.

[4] N. Zhang, M. A. Sirbu and J. M. Peha, "A comparison of migration and multihoming support in IPv6 and XIA", 2017 International Symposium on Networks, Computers and Communications (ISNCC), Marrakech, 2017, pp. 1-8.

[5] Kamaldeep, M. Malik and M. Dutta, "Implementation of single-packet hybrid IP traceback for IPv4 and IPv6 networks", IET Information Security, vol. 12, no. 1,year 2018 pp. 1-6.

[6] D. R. Al-Ani, A. R. Al-Ani, "The Performance of IPv4 and IPv6 in Terms of Routing Protocols using GNS 3 Simulator", Procedia Computer Science, Vol 130, 2018, Pages 1051-1056.

[7] J. Beeharry and B. Nowbutsing, "Forecasting IPv4 exhaustion and IPv6 migration", 2016 IEEE International Conference on Emerging Technologies and Innovative Business Practices for the Transformation of Societies (EmergiTech), Balaclava, 2016, pp. 336-340.

[8] Y. Sookun, and V. Bassoo, "Performance analysis of IPv4/IPv6 transition techniques", 2016 IEEE International Conference on Emerging Technologies and Innovative Business Practices for the Transformation of Societies (EmergiTech), Balaclava, year 2016 pp. 188-193.
[9] Hamarsheh, and Ala, "Deploying IPv4-only Connectivity across Local IPv6-only Access Networks", IETE Technical Review Taylor \& Francis year 2018, pp. 1-14.

[10] F. Siddika, and M. A. Hossen , and S. Saha, "Transition from IPv4 to IPv6 in Bangladesh: The competent and enhanced way to follow", 2017 International Conference on Networking, Systems and Security (NSysS), Dhaka, year 2017, pp. 174-179.

[11] S. Narayan and P. R. Lutui, "Network Performance Evaluation of Jumbo Frames on a Network", 6th International Conference on Emerging Trends in Engineering and Technology, Nagpur, year 2013, pp. 69-72.

[12] Z. Qin, "Seamless converging system for IPv4/IPv6 transition", 2017 9th International Conference on Advanced Infocomm Technology (ICAIT), Chengdu, 2017, pp. 110-113.

[13] S. Aravind and G. Padmavathi, "Migration to IPv6 from IPV4 by dual stack and tunneling techniques", International Conference on Smart Technologies and Management for Computing, Communication, Controls, Energy and Materials (ICSTM), Chennai, year 2015 , pp. 107111.

[14] M. F. Suleiman and J. Cordry, "Analysis of organizations IPv6 deployment strategies in Nigeria and evaluating suitable transition mechanisms," 2017 IEEE 2nd Advanced Information Technology, Electronic and Automation Control Conference (IAEAC), Chongqing, 2017, pp. 695-704.

[15] S. R. Javid and S. K. Dubey, "Implementing OSPFv3 in IPv6 network," 2016 6th International Conference - Cloud System and Big Data Engineering (Confluence), Noida, 2016, pp. 580-584.

[16] C. Udeagha, R. Martin, D. Peck, A. Youton, A. Marshall and J. Clarke, "Migrating from IPV4 to IPV6 in Jamaica", SoutheastCon 2018, St. Petersburg, FL, USA, 2018, pp. 1-8.

[17] D. Chauhan, and S. Sharma, "Performance Evaluation of Different Routing Protocols in IPv4 and IPv6 Networks on the Basis of Packet Sizes”, In Procedia Computer Science, Vol 46, year 2015, pp. $1072-$ 1078.

[18] G. Lencse, and Y. Kadobayashi, "Methodology for the identification of potential security issues of different IPv6 transition technologies: Threat analysis of DNS64 and state full NAT64", Computers \& Security, Vol 77, Year 2018, pp 397-411.

[19] T. Saraj, and M. Yousaf, S. Akbar, A. Qayyum, and M. Tufail, "ISP Independent Architecture (IIA) for IPv6 Packet Traversing and Interconnectivity over Hybrid (IPv4/IPv6) Internet", Procedia Computer Science, Vol 32, year 2014, pp. 973-978. 